\title{
Anomalous non-ergodic scaling in adiabatic multicritical quantum quenches
}

\author{
Shusa Deng, ${ }_{1}^{1}$ Gerardo Ortiz, ${ }^{2}$ and Lorenza Viola ${ }^{1}$ \\ ${ }^{1}$ Department of Physics and Astronomy, Dartmouth College, 6127 Wilder Laboratory, Hanover, New Hampshire 03755, USA \\ ${ }^{2}$ Department of Physics, Indiana University, Bloomington, Indiana 47405, USA
}

(Dated: October 30, 2018)

\begin{abstract}
We investigate non-equilibrium dynamical scaling in adiabatic quench processes across quantum multicritical points. Our analysis shows that the resulting power-law scaling depends sensitively on the control path, and that anomalous critical exponents may emerge depending on the universality class. We argue that the observed anomalous behavior originates in the fact that the dynamical excitation process takes place asymmetrically with respect to the static multicritical point, and that non-critical energy modes may play a dominant role. As a consequence, dynamical scaling requires introducing new non-static exponents.
\end{abstract}

PACS numbers: 73.43.Nq, 75.10.Jm, 64.60.Kw, 05.30.-d

Establishing dynamical scaling relations in many-body systems adiabatically driven out-of-equilibrium across a quantum phase transition has important implications for both condensed-matter physics 1 , and adiabatic quantum computation 2 . A paradigmatic scenario is offered by the so-called Kibble-Zurek scaling (KZS) ${ }^{3.4}$, whereby an homogeneous $d$-dimensional system is linearly driven with a constant speed $1 / \tau$ across an isolated quantum critical point (QCP) described by equilibrium critical exponents $\nu$ and $z$. Under the assumption that, in the thermodynamic limit, the system loses adiabaticity throughout an "impulse region" $\left[t_{c}-\hat{t}, t_{c}+\hat{t}\right]$ which is centered around the QCP and has a characteristic width $2 \hat{t}$, excitations are generated in the final state with a density $n_{\text {ex }}\left(t_{f}\right) \sim \tau^{-d \nu /(\nu z+1)}$. While the KZS and its non-linear generalizations have been verified in several exactly solvable models ${ }^{5}$, departures from the $\mathrm{KZ}$ prediction may occur for more complex quench processes - involving either isolated QCPs in disordered ${ }^{6}$ and infinitely-coordinated systems $\stackrel{7}{\underline{7}}$, or non-isolated QCPs (that is, quantum critical regions) $\stackrel{8,9,10}{ }$. Evidence of non-KZS, however, has also been reported in the apparently simple situation of a quench across a single quantum multicritical point (MCP) in clean spin chains ${ }^{11,12}$.

In this work, we show how multicritical quantum quenches dramatically exemplify the dependence of nonequilibrium scaling upon the control path anticipated in Ref. 9, and demonstrate that anomalous "non-ergodic" scaling may emerge in the thermodynamic limit. While a non-KZS $n_{\mathrm{ex}}\left(t_{f}\right) \sim \tau^{-1 / 6}$ was previously reported ${ }^{11}$ and an explanation given in terms of an "effective dynamical critical exponent" $z_{2}=3$, the meaning of such exponent relied on the applicability of a Landau-Zener (LZ) treatment, preventing general insight to be gained. We argue that the failure of KZS is physically rooted in the shift of the center of the impulse region relative to the static picture, and that $z_{2}$ is determined by the scaling of a path-dependent minimum gap which need not coincide with the critical gap. Furthermore, such a dynamical shift may also cause the contribution from intermediate non-critical energy states to dominate the scaling of the excitation density, via an "effective dimensionality exponent" $d_{2} \neq 0$. We show that the latter leads to the emergence of new scaling behavior $n_{\mathrm{ex}}\left(t_{f}\right) \sim \tau^{-3 / 4}$. A unified understanding is obtained by extending the adiabatic renormalization (AR) approach of Ref. 9 .

Model Hamiltonian.- We focus on the alternating spin-1/2 XY chain described by the Hamiltonian ${ }^{9.13}$ :

$$
H=-\sum_{i=1}^{N}\left(\gamma_{+} \sigma_{x}^{i} \sigma_{x}^{i+1}+\gamma_{-} \sigma_{y}^{i} \sigma_{y}^{i+1}-h_{i} \sigma_{z}^{i}\right),
$$

where $\gamma_{ \pm}=(1 \pm \gamma) / 2, h_{i}=h-(-)^{i} \delta$, and periodic boundary conditions are assumed. Here, $h, \delta \in \mathbb{R}$ are the uniform and alternating magnetic field strength, respectively, whereas $\gamma \in \mathbb{R}$ is the anisotropy (lifting the restriction $\gamma \in[0,1]$ is essential for the present analysis). An exact solution for the energy spectrum of $H$ may be obtained through the steps outlined in Ref. 13. The problem maps into a collection of noninteracting quasi-particle labelled by momentum modes $k \in K_{+}=\{\pi / N, 3 \pi / N, \ldots, \pi / 2-\pi / N\}$, whose excitation gap is given by $\Delta_{k}(\gamma, h, \delta)=4\left[h^{2}+\delta^{2}+\right.$ $\left.\cos ^{2} k+\gamma^{2} \sin ^{2} k-2 \sqrt{h^{2} \cos ^{2} k+\delta^{2}\left(h^{2}+\gamma^{2} \sin ^{2} k\right)}\right]^{1 / 2}$. The quantum phase boundaries are determined by the equations $\mathrm{s}^{9.13} h^{2}=\delta^{2}+1, \delta^{2}=h^{2}+\gamma^{2}$. Thus, the critical lines on the $\gamma=0$ plane consist entirely of MCPs.

Quench dynamics: Exact results.- We assume that the system is initially in the ground state, and that (in the simplest case) a slow quench across a MCP is implemented upon changing a single control parameter according to $\delta \lambda(t)=\lambda(t)-\lambda_{c}=\left|\left(t-t_{c}\right) / \tau\right|^{\alpha} \operatorname{sign}\left(t-t_{c}\right)$ over a time interval $t \in\left[t_{0}, t_{f}\right]$, where $\alpha=1$ corresponds to a linear driving, and $\lambda_{c}$ is the critical value. Thus, the time-dependent Hamiltonian $H(t)$ may be written as: $H(t)=H_{c}+\delta \lambda(t) H_{1}$, where $H_{c}$ is quantum-multicritical at time $t_{c}$ in the thermodynamic limit, and $H_{1}$ is the contribution that couples to the external control (a similar parametrization is possible for quenches involving multiple parameters). Without loss of generality, we may let $t_{c}=0$. In what follows, we shall focus on two representative MCPs, A and B as marked in Fig. 1, each approached through two different paths (path 5 will be 
introduced later) with the following properties:

\begin{tabular}{c|cr|c} 
Path & $\nu$ & $z$ & Quenching scheme \\
\hline 1 & 1 & 2 & $\gamma(t)=\delta(t)=|t / \tau|^{\alpha} \operatorname{sign}(t) ; h=1$ \\
2 & 1 & 2 & $\gamma(t)=|t / \tau|^{\alpha} \operatorname{sign}(t) ; h=1, \delta=1$ \\
3 & $1 / 2$ & 2 & $\gamma(t)=\delta(t)-1=|t / \tau|^{\alpha} \operatorname{sign}(t) ; h=1$ \\
4 & $1 / 2$ & 2 & $\gamma(t)=h(t)-1=|t / \tau|^{\alpha} \operatorname{sign}(t) ; \delta=0$ \\
\hline
\end{tabular}
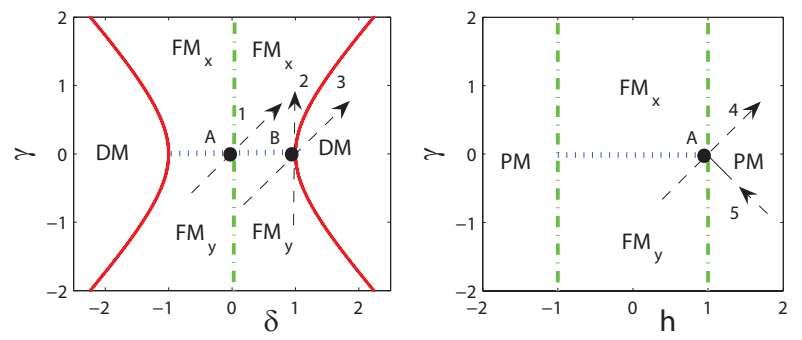

FIG. 1: Phase diagram of $H$ in Eq. (1) when $h=1$ (left) and $\delta=0$ (right). The dashed-dotted (green) line separates the ferromagnetic (FM) and paramagnetic (PM) phases, the solid (red) lines separate dimer (DM) and FM, whereas the dotted (green) line is the superfluid phase (SF). The arrows indicate the control paths we choose to approach the MCPs A and B.

In order to quantify the amount of excitation at a generic instant $t$, we numerically integrate the timedependent Schrödinger equation for $H(t)$ and monitor two standard "non-adiabaticity" indicators ${ }^{7.9 .13}$ : the excitation density, $n_{\mathrm{ex}}$, and the residual energy, $\Delta H$. For a linear quench along either path 1 or 2 (left panel of Fig. 21), we find that $n_{\mathrm{ex}}(t) \sim \tau^{-\nu /(\nu z+1)}=\tau^{-1 / 3}$ and $\Delta H(t) \sim \tau^{-\nu(1+z) /(\nu z+1)}=\tau^{-1}$, which is consistent with $\mathrm{KZS}^{3}$ and our conclusion in Ref. 9. For paths 3 and 4, however (right panel of Fig. 2), we find that $n_{\mathrm{ex}}(t) \sim \tau^{-1 / 6}$ and $\Delta H(t) \sim \tau^{-2 / 3}$, which is non-KZS (in Ref. 11, the $\tau^{-1 / 6}$ scaling was pointed out for an equivalent quench scheme across MCP A). Similar anomalous exponents are found for non-linear quenches along paths 3 or $4, e . g ., n_{\mathrm{ex}}(t) \sim \tau^{-2 / 9}$ for $\alpha=2$.

The above results clearly show that, for quenches across a MCP, whether KZS is obeyed depends sensitively on which control path is chosen. A closer inspection reveals the following important differences: (i) Paths 1,2 start and end in essentially the same phase, correspondingly the excitation spectrum is invariant under a transformation $\lambda \mapsto-\lambda$ of the control parameters. Paths 3,4 do not exhibit this symmetry; (ii) Along paths 3,4 , the MCPs A and B belong to the Lifshitz universality class $(\nu=1 / 2)$, although all paths share $z=2$. It is then natural to ask which of these differences may play a role in determining the anomalous dynamical scaling behavior. To answer this question, we introduce another path across MCP A (path 5), $h(t)=1+|\gamma(t)|=1+|t / \tau|$, which starts and ends in the PM phase but, in each of the two segments, crosses the MCP A with Lifshitz exponents. Surprisingly, the observed scaling is $n_{\mathrm{ex}}(t) \sim \tau^{-3 / 4}$ (left panel of Fig. 3), which is neither KZS nor $-1 / 6$. An identical $-3 / 4$ scaling holds for a "V-shaped" path across
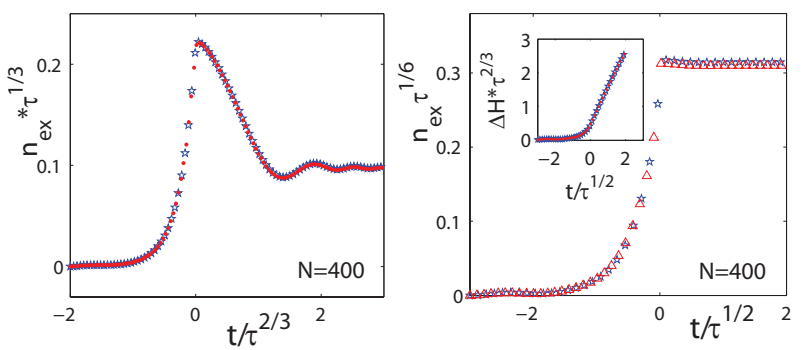

FIG. 2: Exact scaling of the excitation density throughout a linear quench along path 2 (left) and path 3 (right). Right inset: Scaling of the residual energy along path 3 .
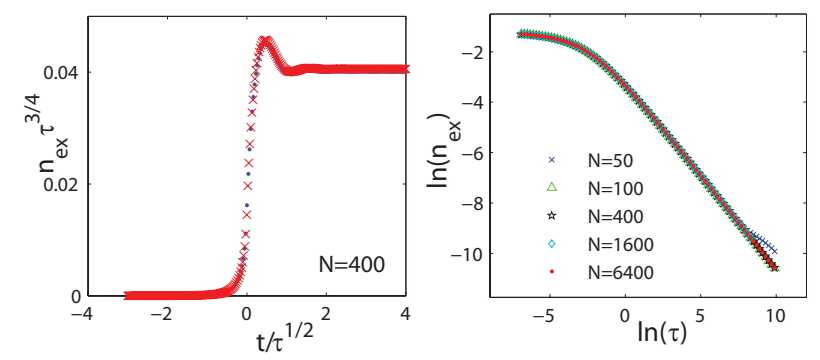

FIG. 3: Left: Exact scaling of the excitation density throughout a linear quench along path 5. Right: Scaling of the final excitation density in path 5 for different system size: $n_{\mathrm{ex}}\left(t_{f}\right)$ is the same to a numerical accuracy of $10^{-6}$, up to $\tau<2 \times 10^{5}$. A linear fit yields $-0.747 \pm 0.001$ over the range $200<\tau<2000$.

MCP B, that starts and ends in the DM phase. As finitesize analysis reveals, all the observed anomalous scalings are practically independent upon system size over a wide range of quench rates (see e.g. right panel of Fig. 3), establishing them as truly thermodynamic in nature 14 .

Landau-Zener analysis.- We begin to seek an understanding from limiting cases where an exact solution for $n_{\mathrm{ex}}\left(t_{f}\right)$ may be obtained based on the LZ picture $\underline{15}$. This is possible provided that $\alpha=1$ and the Hamiltonian can be decoupled into effective two-level systems. Among the above-mentioned paths, only paths 4 and 5 (for which $\delta=0$ ) can be exactly mapped to a LZ problem, thanks to the possibility of rewriting $H$ in Eq. (11) as $H=\sum_{k} \hat{H}_{k}=\sum_{k} B_{k}^{\dagger} H_{k} B_{k}$, where $B_{k}^{\dagger}=\left(c_{-k}, c_{k}^{\dagger}\right)$ and

$$
H_{k}=\left(\begin{array}{cc}
H_{k, 11} & H_{k, 12} \\
H_{k, 12}^{*} & -H_{k, 11}
\end{array}\right)=2\left(\begin{array}{cc}
-h+\cos k & \gamma \sin k \\
\gamma \sin k & h-\cos k
\end{array}\right) \text {. }
$$

A rotation $R_{k}\left(q_{k}\right), q_{k} \in[-\pi / 2, \pi / 2)$, renders the offdiagonal terms in Eq. (2) independent upon $\gamma$ (hence time), allowing use of the LZ formula. Consider path 4 first. By choosing $\tan 2 q_{k}=-\sin k$, the transformed Hamiltonian matrix elements become $H_{k, 11}^{\prime}=-2(1-$ $\cos k) \cos 2 q_{k}-2 t / \tau\left(\cos 2 q_{k}-\sin k \sin 2 q_{k}\right)$, and $H_{k, 12}^{\prime}=$ $2(1-\cos k) \sin 2 q_{k}$. If the critical mode $k_{c}$ is defined by requiring $\Delta_{k_{c}}=0$ in the thermodynamic limit, we have $k_{c}=0$ for the MCP A. We may then let $\tan 2 q_{k} \approx \sin 2 q_{k}$, and the appropriate $q_{k} \approx-k / 2$. From the LZ formula, 
the asymptotic $\left(t_{f} \rightarrow \infty\right)$ excitation probability reads

$p_{k}=e^{-2 \pi \tau(1-\cos k)^{2} \sin ^{2} 2 q_{k} /\left(\cos 2 q_{k}-\sin k \sin 2 q_{k}\right)} \approx e^{-\pi \tau k^{6} / 2}$,

where the approximation follows from a Taylor expansion around $k_{c}$. Integrating over all modes yields $n_{\mathrm{ex}}\left(t_{f}\right) \sim$ $\tau^{-1 / 6}$, which is consistent with our exact numerical result. Therefore, mathematically, the $\tau^{-1 / 6}$ scaling follows from the fact that the exponent in $p_{k}$ scales as $k^{6}=k^{2 z_{2}}$, with $z_{2}=3$. In turn, this originates from the scaling of the off-diagonal terms $H_{k, 12}^{\prime} \sim k^{z_{2}}$. Physically, as we shall later see by invoking AR, $H_{k, 12}^{\prime}$ may be interpreted as the minimum gap for mode $k$ along path 4 .

To unveil the $\tau^{-3 / 4}$ scaling, it is necessary to use the exact finite-time LZ solution. For simplicity, we restrict to half of path 5, by quenching the system from the PM phase up to the MCP A. This has the benefit of avoiding the non-analytic time-dependence of the control parameters that path 5 exhibits at $\mathrm{A}$, while leaving the $\tau^{-3 / 4}$ scaling unchanged thanks to the symmetry of the excitation spectrum. Starting from Vitanov's expression [Eq. (7) in Ref. 16], the excitation probability $p_{k}\left(t_{f}\right)$ can be computed via the parabolic cylinder function $D_{v}(z)$,

$$
\begin{aligned}
p_{k}\left(t_{f}\right) & =e^{-\pi \omega^{2} / 4} \mid D_{i \omega^{2} / 2}\left(T_{f} \sqrt{2} e^{i 3 \pi / 4}\right) \cos \theta\left(T_{f}\right) \\
& \left.-\frac{\omega}{\sqrt{2}} e^{-i \pi / 4} D_{i \omega^{2} / 2-1}\left(T_{f} \sqrt{2} e^{i 3 \pi / 4}\right) \sin \theta\left(T_{f}\right)\right)\left.\right|^{2},
\end{aligned}
$$

where $\omega=(1-\cos k) \sin 2 q_{k} \sqrt{\tau} / \sqrt{\cos 2 q_{k}+\sin 2 q_{k} \sin k} \sim$ $k^{3} \sqrt{\tau}$ is the rescaled coupling strength, $T_{f}=-\omega / \sin k \sim$ $-k^{2} \sqrt{\tau}$ is the rescaled time, $\tan 2 q_{k}=\sin k$, and $\theta\left(T_{f}\right)=1 / 2 \arctan \left(\omega / T_{f}\right)+\pi / 2$. Since for our quench process $\omega \ll\left|T_{f}\right| \ll 1$ around $k_{c}$, we may estimate $p_{k}\left(t_{f}\right)$ by Taylor-expanding $D_{v}(z)$ around $T_{f}=0$ :

$$
\begin{aligned}
p_{k}\left(t_{f}\right) & \approx\left(1-e^{-\pi \omega^{2} / 2}\right) / 2+\cos ^{2} \theta\left(T_{f}\right) e^{-\pi \omega^{2} / 2} \\
& -\sin 2 \theta\left(T_{f}\right) / 2 \sin \chi_{k} \sqrt{1-e^{-\pi \omega^{2}}}
\end{aligned}
$$

where $\chi_{k} \approx \pi / 4$ around $k_{c}$. This approximation breaks when $T_{f} \sim 1$, setting the scaling of the highestmomentum contributing mode, that is, $k_{\max } \sim \tau^{-1 / 4}$. In Eq. (3), the dominant term $\cos ^{2} \theta\left(T_{f}\right) e^{-\pi \omega^{2} / 2} \sim$ $\cos ^{2} \theta\left(T_{f}\right) \sim\left|\omega / T_{f}\right|^{2} \sim k^{2}$ since $e^{-\pi \omega^{2} / 2} \approx 1$ within $k_{\max }$, which means $p_{k}\left(t_{f}\right) \sim k^{2}$. Thus, $n_{\mathrm{ex}}\left(t_{f}\right)=$ $\int_{0}^{k_{\max }} p_{k}\left(t_{f}\right) \sim k_{\max }^{3} \sim \tau^{-3 / 4}$, in agreement with our numerical results. Remarkably, the fact that $p_{k}\left(t_{f}\right) \sim$ $\left(k-k_{c}\right)^{d_{2}}, d_{2}=2$, indicates that $k_{c}$ is not excited despite a static QCP being crossed, and also that the excitation is dominated by intermediate energy states. In fact, at the MCP A, the modes around $k_{c}$ are still far from the impulse region, since $\left|T_{f}\right| \gg \omega$, which sets the LZ transition time scale ${ }^{16}$. This is in stark contrast with the main assumption underlying $\mathrm{KZS}$, where the center of the impulse region is the static QCP, and excitations are dominated by modes near $k_{c}$, as reflected in the typical scaling $p_{k} \sim\left(k-k_{c}\right)^{0}$. Therefore, the shift of the actual
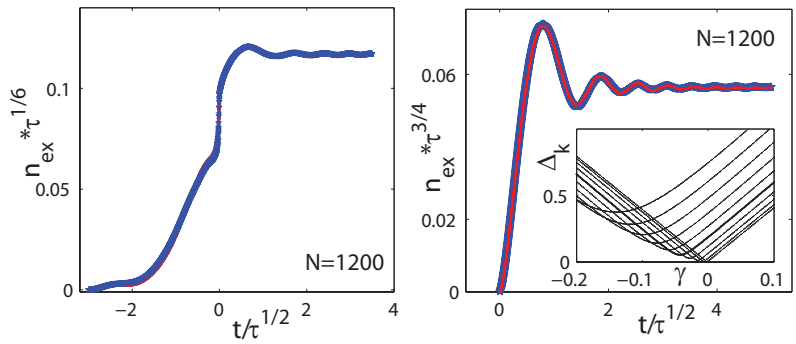

FIG. 4: Scaling of the excitation density from first-order AR for a linear quench along path 3 (left) and half- 5 starting at MCP A (right). Right inset: Low-lying single-mode excitation spectrum along path 4 for $N=100$.

(dynamical) impulse region relative to the static one is ultimately responsible for the anomalous $\tau^{-3 / 4}$ scaling.

Perturbative scaling approach.- Since the system becomes gapless at a single MCP along all the paths under study, first-order AR is a viable approach 4.9 . Let $\left|\psi_{m}(t)\right\rangle$ be a basis of snapshot eigenstates of $H(t)$, with snapshot eigenvalues $E_{m}(t), m=0$ labelling the ground state. The time-evolved state may be expanded as $|\psi(t)\rangle=c_{0}^{(1)}(t)\left|\psi_{0}(t)\right\rangle+\sum_{m \neq 0} c_{m}^{(1)}(t)\left|\psi_{m}(t)\right\rangle$, where the coefficients $c_{m}^{(1)}(t)$ determine the excitation amplitudes and are given by Eq. (4) in Ref. 9. First-order AR calculations of $n_{\mathrm{ex}}(t)$ demonstrate that for linear quenches along paths 1 and $2, n_{\mathrm{ex}} \sim \tau^{-1 / 3}$, whereas $n_{\mathrm{ex}} \sim \tau^{-1 / 6}$ along paths 3 and 4 (left panel of Fig. 4). Since the nonanalyticity at $\mathrm{A}$ in path 5 might cause problems in AR, again we choose to study half of path 5 (right panel of Fig. 41). All the AR results agree with the exact simulation results, confirming that $\mathrm{AR}$ reproduces the correct dynamical scaling across a generic isolated QCP.

Predicting the scaling exponent based on AR requires scaling assumptions for the contributions entering $c_{m}^{(1)}(t)$ [i.e., $\Delta_{m}(t)=E_{m}(t)-E_{0}(t)$ and $\left.\left\langle\psi_{m}(t)\left|H_{1}\right| \psi_{0}(t)\right\rangle\right]$ and the ability to change discrete sums of all the contributing excited states into integrals, for which the density of excited states $\rho(E)$ is required. Since typically the AR prediction is consistent with KZS, anomalous behavior must stem from anomalous scaling assumptions of (one or more of) these ingredients. We first examine the excitation spectrum along different paths. Notice that since $H_{1}$ is a one-body perturbation, only single-mode excitations are relevant, thus the index $m$ labeling many-body excitations may be identified with a momentum mode. Along paths 1 and 2, it turns out that the minimum gap among all modes is always located at $k_{c}$, whereas along paths 3 and 4 , the minimum gap is located at $k_{c}$ only at the MCP. This suggests that knowing the critical exponents of the MCP alone need not suffice to determine the dynamical scaling due to the existence of "quasi-critical" modes along paths 3 and 4 . In fact, mathematically, along path $4, \partial \Delta_{k}(\gamma, 1+\gamma, 0) / \partial \gamma=0$ gives the location of the minimum gap for each mode $k$ at $\tilde{\gamma}=(\cos k-1) /\left(1+\sin k^{2}\right)$, which is largely shifted into the FM phase (see inset in 
Fig. 4). By inserting this relation back into $\Delta_{k}$, the composite function $\Delta_{k}(\tilde{\gamma}) \equiv \tilde{\Delta}_{k} \sim\left(k-k_{c}\right)^{3}$. Following the same procedure also yields $\tilde{\Delta}_{k} \sim\left(k-k_{c}\right)^{3}$ along path 3 , while $\tilde{\Delta}_{k}$ has the same scaling as $\Delta_{k}$ at the MCP along paths 1 and 2. This motivates modifying the AR scaling assumptions of Ref. 9 as follows: $E_{m}(t)-E_{0}(t)=\delta \lambda(t)^{\nu z} f_{m}\left(\Delta_{m}\left(t_{\min }\right) / \delta \lambda(t)^{\nu z}\right)$, where $\Delta_{m}\left(t_{\text {min }}\right)$ is the minimum gap of mode $m$ attained at $t_{\text {min }}$ along the path, and $f_{m}$ is a scaling function.

The above modification requires the scaling of $\rho(E)$ to be modified by letting $\rho(E) \sim E^{d / z_{2}-1}$, where $z_{2}$ comes from the dispersion relation of $\Delta_{m}\left(t_{\min }\right)$. If the minimum gap of any mode is below a certain energy along the path, that mode should be counted into the contributing excited states. Accordingly, we have $z_{2}=z=2$ along paths 1,2 , half- 5 , and $z_{2}=3$ along paths 3,4 . Back to the LZ analysis, note that the off-diagonal term $H_{12}^{\prime}(k)$ $i s$ the minimum gap of mode $k$ along the path if there exists a time at which the diagonal term $H_{11}^{\prime}(k)=0$, as it happens for path 4 . For path 5, however, the offdiagonal term never becomes the minimum gap since the system never leaves the PM phase. Therefore, the offdiagonal term in the LZ picture need not suffice to determine the dynamical scaling, and the shift of the location of the minimum gap for each mode from the static QCP is at the root of the anomalous behavior we observe. Lastly, we consider the matrix elements of $H_{1}$. Numerical simulations suggest that $\left\langle\psi_{m}(t)\left|H_{1}\right| \psi_{0}(t)\right\rangle=$ $\delta \lambda(t)^{\nu z-1} g_{m}\left(\Delta_{m}\left(t_{\min }\right) / \delta \lambda(t)^{\nu z}\right)$, where $g_{m}$ is a scaling function, and $\Delta_{m}\left(t_{\min }\right)$ is the minimum gap of mode $m$ along a path that extends the actual path to $t_{f} \rightarrow \infty$ when the quench is stopped at the MCP, and coincides with the actual path otherwise. Then along paths 1 and $2, \Delta_{m}\left(t_{\min }\right) \sim k^{2}$, whereas along paths 3,4 , and half- 5 , $\Delta_{m}\left(t_{\text {min }}\right) \sim k^{3}$. Together with the other scaling assump- tions, and taking the linear case $\alpha=1$ as an example, AR yields $\left|c_{m}^{(1)}\right| \sim k^{0}, n_{\mathrm{ex}} \sim \tau^{-\left(z / z_{2}\right)(\nu /(\nu z+1))}$ along paths 1 to 4 , and $\left|c_{m}^{(1)}\right| \sim k^{1}, n_{\mathrm{ex}} \sim \tau^{-3 \nu /(\nu z+1)}$ along half-5 path, which completely agrees with the numerical results.

Building on the above analysis, we argue on physical grounds that the scaling of the excitation density for quenches across an arbitrary (standard or multicritical) isolated QCP is determined by three conditions: (i) From the condition of adiabaticity breaking, the typical gap, $\hat{\Delta}$, scales as $\hat{\Delta} \sim \tau^{-\alpha \nu z /(\alpha \nu z+1)}$; (ii) An accessible excited state contributes to the excitation if and only if its minimum gap along the path matches with this typical gap, $\tilde{\Delta}_{k} \sim \hat{\Delta}$, with $\tilde{\Delta}_{k} \sim\left(k-k_{c}\right)^{z_{2}}$; (iii) The excitation probability, $p_{k}$, scales as $p_{k} \sim\left(k-k_{c}\right)^{d_{2}}$, where $d_{2}$ can differ from 0 if the center of the impulse region is greatly shifted relative to the static limit. Then upon integrating up to energy $\hat{\Delta}$, and using $p_{E} \sim p_{k(E)} \sim E^{d_{2} / z_{2}}$, yields

$$
n_{\mathrm{ex}} \sim \hat{\Delta}^{\left(d+d_{2}\right) / z_{2}} \sim \tau^{-\left(d+d_{2}\right) \alpha \nu z /\left[z_{2}(\alpha \nu z+1)\right]},
$$

which is consistent with all the results found thus far. While KZS corresponds to $z_{2}=z, d_{2}=0$, situations where $z_{2} \neq z$ and/or $d_{2} \neq 0$ are genuinely dynamical: knowledge about the path-dependent excitation process becomes crucial and non-equilibrium exponents cannot be fully predicted from equilibrium ones. Interestingly, in the model under examination the Lifshitz universality class appears to be the only universality class for which anomalous scaling occurs, among all possible paths involving MCPs. Whether Lifshitz behavior may constitute a sufficient condition for anomalous behavior requires further investigation in other many-body systems.

It is a pleasure to thank Tommaso Caneva for insightful exchange. S.D. gratefully acknowledges support from a Hull Graduate Fellowship.
1 S. Sachdev, Quantum Phase Transitions (Cambridge University Press, Cambridge, 1999).

2 E. Fahri et al., Science 292, 472 (2001); G. E. Santoro and E. Tosatti, J. Phys. A 39, R393 (2006).

3 W. H. Zurek, U. Dorner, and P. Zoller, Phys. Rev. Lett. 95, 105701 (2005); J. Dziarmaga, ibid. 95, 245701 (2005); B. Damski and W. H. Zurek, Phys. Rev. A 73, 063405 (2006); F. M. Cucchietti et al., ibid. 75, 023603 (2007).

4 A. Polkovnikov, Phys. Rev. B 72, 161201 (2005); A. Polkovnikov, and V. Gritsev, Nat. Phys. 4, 477 (2008).

${ }^{5}$ R. W. Cherng and L. S. Levitov, Phys. Rev. A 73, 043614 (2006); A. Fubini, G. Falci, and A. Osterloh, New J. Phys. 9, 134 (2007); V. Mukherjee, A. Dutta, and D. Sen, Phys. Rev. B 77, 214427 (2008); S. Mondal, D. Sen, and K. Sengupta, Phys. Rev. B 78, 045101 (2008); D. Sen, K. Sengupta, and S. Mondal, Phys. Rev. Lett. 101, 016806 (2008); R. Barankov, and A. Polkovnikov, Phys. Rev. Lett. 101, 076801 (2008).

6 J. Dziarmaga, Phys. Rev. B 74, 064416 (2006); T. Caneva, R. Fazio, and G. E. Santoro, Phys. Rev. B 76, 144427 (2007).
7 T. Caneva, R. Fazio, and G. E. Santoro, Phys. Rev. B 78, 104426 (2008).

${ }^{8}$ F. Pellegrini, et al., Phys. Rev. B 77, 140404 (2008).

9 S. Deng, G. Ortiz, and L. Viola, Europhys. Lett. 84, 67008 (2008).

10 D. Chowdhury, U. Divakaran, and A. Dutta, arXiv: 0906.1161 .

11 V. Mukherjee, U. Divakaran, A. Dutta, and D. Sen, Phys. Rev. B 76, 174303 (2007); U. Divakaran, V. Mukherjee, A. Dutta, and D. Sen, J. Stat. Mech. P02007 (2009).

12 S. Mondal, K. Sengupta, and D. Sen, Phys. Rev. B 79, 045128 (2009).

13 S. Deng, L. Viola, and G. Ortiz, Recent Progress in ManyBody Theories, Vol. 11 (World Scientific, Singapore, 2008), p. 387, arXiv:0802.3941

14 Since $\nu z<z$, to leading order the scaling of $\Delta_{k}$ is dominated by the control parameter rather than by the size.

15 C. Zener, Proc. R. Soc. London A 137, 696 (1932).

16 N. V. Vitanov, Phys. Rev. A 59, 988 (1999). 